\title{
Ultrasound-induced encapsulated microbubble phenomena
}

\author{
M. Postema $* \dagger$, A. van Wamel $* \dagger$, C.T. Lancée $*$, N. de Jong $* \dagger \ddagger$
}

* Dept. of Experimental Echocardiography, Thoraxcentre, Erasmus MC, Rotterdam, The Netherlands; † Interuniversity Cardiology Institute of The Netherlands, Utrecht, The Netherlands; and $\ddagger$ Physics of Fluids, Department of Applied Physics, Faculty of Science and Technology, University of Twente, Enschede, The Netherlands

corresponding author:

Michiel Postema, Dept. of Experimental Echocardiography, Thoraxcentre, Erasmus MC, Dr. Molewaterplein 50, Room Ee-23.02, zip code 3015 GE, Rotterdam, The Netherlands tel: +31.10.4089358, fax: +31.10.4089445, e-mail: m.postema@erasmusmc.nl

Running title: Encapsulated microbubble phenomena 


\section{Abstract}

When encapsulated microbubbles are subjected to high-amplitude ultrasound, the following phenomena have been reported: oscillation, translation, coalescence, fragmentation, sonic cracking, and jetting.

In this paper, we explain these phenomena, based on theories that were validated for relatively big, free (not encapsulated) gas bubbles. These theories are compared to high-speed optical observations of insonified contrast agent microbubbles. Furthermore, the potential clinical applications of the bubble-ultrasound interaction are explored.

We conclude that most of the results obtained are consistent with free gas bubble theory. Similar to cavitation theory, the number of fragments after bubble fission is in agreement with the dominant spherical harmonical oscillation mode. Remarkable are our observations of jetting through contrast agent microbubbles. This is high enough to penetrate any human cell. Hence, liquid jets may act as remote-controlled microsyringes, delivering a drug to a region of interest.

Encapsulated microbubbles have (potential) clinical applications in both diagnostics and therapeutics. 


\section{Key words}

Encapsulated microbubbles, ultrasound contrast agent, radiation forces, coalescence, fragmentation, jets.

\section{Introduction}

Blood does not reflect ultrasound well, but its response may be enhanced by gas microbubbles of a few micrometers in diameter that are introduced into the blood pool (Goldberg et al., 2001). These microbubbles oscillate upon insonification, and generate as such a characteristic acoustic response.

An ultrasound contrast agent (UCA) is a liquid containing gas microbubbles which are encapsulated by a shell. UCAs are widely used in clinical diagnostic imaging (Becher and Burns, 2000; Goldberg et al., 2001). The intravascular use of UCA enhances the quantification of perfusion and blood flow (Klibanov, 1999; Wei, 2001), and lowers the detectability threshold of tumor and tissue vascularization, using conventional Doppler instruments (Burns et al., 1990). An overview of UCA detection procedures in ultrasound imaging has been presented by de Jong et al. (2000b), and an overview of UCA imaging innovations has been put forward by Chiou et al. (2000).

To understand encapsulated microbubble behavior better in order to develop or enhance applications in diagnostics and therapeutics, UCA microbubbles have 
been subjected to high-speed photography. Postema et al. (2003a) gave an overview of publications on high-speed optical observations of insonified UCA.

Observations of dynamic UCA microbubble behavior have resulted in new insights and novel approaches in diagnostics and therapeutics: The nonlinear behavior of oscillating bubbles has led to the development of contrast harmonic imaging (Schrope and Newhouse, 1993; de Jong et al., 1994, 2002; Bouakaz et al., 2002a,b), whereas observations of bubble destruction have led to the development of high-MI imaging techniques (Uhlendorf and Hoffmann, 1994; Frinking et al., 2001).

Targeted UCA delivery is based on microbubbles with ligands attached to them (Klibanov, 1999; Unger et al., 2001). These bubbles circulate through vessels and accumulate at a target tissue, thereby marking the target in ultrasound images. Klibanov (1999) put forward that targeted microbubbles may be applied for selective delivery to the areas where selective enhancement of the action of ultrasound would be required, and if such a bubble would contain plasmid DNA, enzyme, or another therapeutic agent, it might be released at the site of interest during insonification. Another means of targeting was suggested by Dayton et al. (2001b). They provided optical verification that microbubbles oscillate differently when inside neutrophils in response to insonification, and emit acoustic signals that are distinct from free microbubbles. Thus, microbubbles within neutrophils might be detected, providing a potential method to image activated neutrophils as 
they accumulate at sites of inflammation.

Recently, Unger et al. (2002) gave an overview of the therapeutic applications of UCA microbubbles. They presented experimental results using UCAs for thrombolysis, drug delivery, and gene delivery. Other recent advances in local drug and gene delivery include the in vivo delivery of a virus vector, attached to albumin microbubbles (Shohet et al., 2000), the in vitro and in vivo transfection of endothelial cells with plasmid DNA mixed with UCA (Taniyama et al., 2002), and the accelerated cellular drug uptake in vitro when UCA microbubbles are present (van Wamel et al., 2002). Tachibana et al. (1999) subjected cells in the presence of a photosensitive drug to continuous ultrasound, and noticed surface pores on the cells. Inducing porosities in cells with ultrasound, generally referred to as sonoporation, has potential applications in (tumor) cell lysis and selective delivery of drugs and genes into cells. Independently, it was demonstrated that moderate microbubble oscillations are sufficient to achieve rupture of lipid membranes, in a regime in which the bubble dynamics can be accurately controlled (Marmottant and Hilgenfeldt, 2003). This might enable the transport of drugs through the cell membrane.

At high acoustic amplitudes, destructive effects of UCA microbubbles have been observed, such as hemorrhaging (Skyba et al., 1998; Maruvada and Hynynen, 2002), and lysis (van Wamel et al., 2002). Kudo et al. (2002) performed an in vitro study on bovine arterial cell damage by ultrasound in combination with 
microbubbles. They found that the locations of damaged cells were similar to the locations where violent bubble collapse was observed, suggesting that bubble collapse is responsible for cell membrane damage.

In this paper, we present an overview of dynamic encapsulated microbubble behavior observed at high acoustic amplitudes. The mechanisms for each type of behavior, found in literature on free (not encapsulated) gas bubbles, acoustics, and cavitation, are correlated to our high-speed optical observations. Furthermore, we address the potential clinical applications of the phenomena.

Previously, some UCA microbubble destruction phenomena were categorized by Chomas et al. (2001a) and Postema et al. (2002a). From our optical observations, we discriminate the following categories: oscillation, translation, coalescence, fragmentation, sonic cracking, and jetting.

In the following subsections, we give a brief overview of these phenomena, and an explanation of their occurrence. Most theories on bubble behavior were validated for big, free (not encapsulated) microbubbles.

\section{Oscillation}

When a microbubble is exposed to an oscillating acoustic signal, it undergoes alternate expansions and contractions with the same amplitude and duration at low driving pressures (Strasberg, 1956). This regime of pulsation amplitudes has been referred to as moderate (Vokurka, 1986). Bubble activity which may 
occur at relatively low-amplitude pressures has been denoted as stable cavitation (Miller and Nyborg, 1983). As the driving pressure increases, more complex nonlinear interactions occur: greater bubble expansion amplitude than contraction amplitude, and relatively slow expansion followed by rapid contraction (collapse). This behavior has been referred to as violent (Vokurka, 1986) or inertial (or transient) cavitation (Miller and Nyborg, 1983; Flynn and Church, 1988; Barnett, 1998). It has been associated with the production of harmonic signals (Postema et al., 2003a).

The transition from the moderate to the violent regime is rather abrupt: For any driving pressure, there exists a transitional equilibrium microbubble radius $R_{0}^{\mathrm{tr}}$, above which microbubbles pulsate like inertial cavitaties (Flynn, 1975; Flynn and Church, 1988; Hilgenfeldt et al., 1998). This transition is referred to as the cavitation threshold. A bubble is judged to have grown into an inertial cavity when its maximum radius is greater than approximately twice its equilibrium radius (Flynn, 1975; Flynn and Church, 1988).

A number of models for computing radius-time curves of insonified gas bubbles has been developed. These models differ both in complexity and in the range of acoustic amplitudes for which they can be used (Vokurka, 1986).

To model the behavior of UCA microbubbles, the presence of an encapsulation around the gas core has to be accounted for. Hence, we have to introduce parameters that describe the dynamic behavior of the shell. 
Presently, two models have been used for modeling UCA microbubble oscillations. de Jong et al. (1994) used a modified RPNNP equation, named after its developers Rayleigh, Plesset, Neppiras, Noltingk, and Poritsky. They accounted for the presence of a shell by introducing a shell stiffness and a shell friction parameter. Morgan et al. (2000) used a modified Herring equation. They introduced the shell properties thickness, elasticity modulus, and viscosity. Both models are consistent with optical observations at low driving pressures (de Jong et al., 2000a; Morgan et al., 2000; Postema et al., 2003a). Currently, models on UCA microbubble oscillating behavior at high driving pressures have been under investigation (Stride and Saffari, 2003).

\section{Translation}

Translations of UCA microbubbles in the direction of the sound field and towards each other have been frequently observed with high-speed cameras (Dayton et al., 1996, 1997b, 1999, 2001a; Postema et al., 2003c). Microbubble translation in the direction of the sound field has been attributed to a primary radiation force resulting from a pressure gradient across the bubble surface. The translation is maximal in contraction phase (Reddy and Szeri, 2002). It was demonstrated that the velocity $v$ of a bubble in a steady fluid subjected to an ultrasound field can be 
expressed by the following ordinary differential equation (Tortoli et al., 2001):

$$
m \frac{\mathrm{d} v}{\mathrm{~d} t}=F_{\mathrm{rad}}+F_{\mathrm{d}}
$$

where $F_{\text {rad }}$ is the primary radiation force, $F_{\mathrm{d}}$ is the drag force, $m \approx \frac{2 \pi}{3} \rho R_{0}^{3}$ is the mass of the translating bubble, equivalent to half the mass of the displaced surrounding fluid (Leighton, 1994), in which $R_{0}$ is the ambient bubble radius, and $\rho$ is the density of the surrounding fluid. The primary radiation force is given by (Tortoli et al., 2001):

$$
F_{\mathrm{rad}}=\frac{p_{\mathrm{ac}}^{2} R_{0}}{\rho c f} \frac{\delta_{\mathrm{t}} \frac{f \mathrm{r}}{f}}{\left[\left(\frac{f \mathrm{r}}{f}\right)^{2}-1\right]^{2}+\left(\delta_{\mathrm{t}} \frac{f \mathrm{r}}{f}\right)^{2}},
$$

where $c$ is the speed of sound, $p_{\mathrm{ac}}$ is the peak rarefactional acoustic pressure, $\delta_{\mathrm{t}}$ is the dimensionless total damping coefficient (Medwin, 1977), and $f_{\mathrm{r}}$ is the bubble resonant frequency (Medwin, 1977). The drag force is given by (Tortoli et al., 2001):

$$
F_{\mathrm{d}}=-\frac{\pi \eta}{4} C_{\mathrm{d}} \operatorname{Re} R_{0} v(t)
$$

where $\eta$ is the shear viscosity of the fluid, $\operatorname{Re}=\frac{2 \rho R_{0}}{\eta}|v(t)|$ represents the Reynolds number, and $C_{\mathrm{d}}=\frac{24}{\mathrm{Re}}+\frac{6}{1+\sqrt{\mathrm{Re}}}+0.4$ is the drag coefficient.

The microbubble translations towards each other have been attributed to secondary radiation forces: oscillating bubbles generate spatially varying pressure fields. If two bubbles are either both below or both above the resonant size, this results in attraction. However, if one bubble is below and the other is above the 
resonant size, they oscillate out of phase (Pain, 1992; Leighton, 1994; Reddy and Szeri, 2002), causing the bubbles to recede from each other. The mean approach velocity $v_{\mathrm{a}}$ of two identical bubbles is given by Dayton et al. (1997b):

$$
v_{\mathrm{a}}=-\frac{\left(2 \pi f p_{\mathrm{ac}}\right)^{2}}{27 \eta} \rho \kappa^{2} \frac{R_{0}^{5}}{d_{0}^{2}},
$$

where $\kappa$ is the compressibility of the bubble, and $d_{0}$ is the initial distance between the centers of the two bubbles.

\section{Coalescence}

Ultrasound-induced microbubble coalescence is the fusion of two or more microbubbles. As adjacent bubbles expand, the pressure in the film between them increases, resulting in a deformation (flattening) of the bubble surfaces. The continuing bubble expansion causes drainage of the interposed film. This thinning continues until a critical thickness below $0.1 \mu \mathrm{m}$ is reached, at which the Van der Waals attractive forces results in film rupture and the coalescence of the bubbles (Duineveld, 1994). Thinning and rupture of thin liquid films have been described by Sheludko (1967), Kralchevsky et al. (1996), and Narsimhan and Ruckenstein (1996).

Flattening of the adjacent bubble surfaces occurs if and only if the bubble system has a Weber number We $>0.5$ (Chesters and Hoffmann, 1982). The Weber number for a fluid containing two bubbles with radii $R_{1}$ and $R_{2}$, 
respectively, is given by the inertial force relative to the surface tension force:

$$
\mathrm{We}=\frac{\rho u^{2}}{\frac{\sigma}{R_{\mathrm{m}}}},
$$

where $u$ is the relative approach velocity of the bubble walls: $u \approx \dot{R}_{1}+\dot{R}_{2}, \rho$ is the fluid density, $\sigma$ is the surface tension, and $R_{\mathrm{m}}$ is the mean bubble radius for which holds: $\frac{2}{R_{\mathrm{m}}}=\frac{1}{R_{1}}+\frac{1}{R_{2}}$. In our results, Weber numbers are relatively high because of the rapid bubble expansions, with maximal radius increases of several $\mathrm{m} \mathrm{s}^{-1}$. If the Weber number is lower than 0.5 , bubble coalescence will always occur, without flattening of the adjacent surfaces prior to contact (Chesters and Hoffmann, 1982).

In a separate paper, we investigated the coalescence mechanism of microbubbles, based on high-speed optical observations of insonified UCA (Postema et al., 2003c). We investigated the mechanism of film drainage by comparing the observed coalescence times with calculated film drainage times from the Reynolds equation (6). The Reynolds drainage time $\tau_{\mathrm{d}}$ of the interposed film until a critical thickness $h_{\mathrm{c}}$ is given by:

$$
\tau_{\mathrm{d}}=\frac{3 \eta R_{\mathrm{f}}^{2} R_{\mathrm{m}}}{8 \sigma h_{\mathrm{c}}^{2}}
$$

where $R_{\mathrm{f}}$ is the film radius, and $R_{\mathrm{m}}$ is the mean bubble radius.

The calculated drainage times prove too long to be compatible with the observations. Stated differently, if the film between bubbles thinned by Reynolds drainage alone during the observed time span, it would only reach a minimum 
thickness $h_{\mathrm{m}}$ that is much larger than that needed to induce film rupture. Hence, a mechanism for film rupture must be at work that overrules the drainage. We suggest that shape instabilities of the bubble can cause local corrugations that bridge the film and rupture it, before thinning of the whole film. We show that small perturbations on the bubble surface may grow tremendously, a phenomenon known as parametric instability. The initial perturbations may come from thermal undulations and external radiation forces. We show that the latter are dominant, and lead to sufficiently large undulations to bridge the minimum thickness $h_{\mathrm{m}}$ within half an ultrasonic cycle.

Bounce is the process where bubbles approach and flatten, but do not coalesce (Postema et al., 2003c). This behavior has been attributed to the drainage given in eq. (6) taking more time than the expansion phase.

\section{Fragmentation}

Fragmentation is the fission of a bubble into smaller bubbles (Postema et al., 2002a). Although acoustical observations have been associated with microbubble destruction (Shi et al., 2000), fragmentation of UCA microbubbles was first visualized with high-speed cameras in 2001 (Chomas et al., 2001a; Postema et al., 2001). In the same year, Brennen (2001) computed the number of fragments for a cavitation bubble distorted by spherical harmonics. He demonstrated that for

any positive $\Gamma=\frac{\rho R^{2} \ddot{R}}{\sigma}$, where $\ddot{R}$ denotes the second time derivative of $R$, there 
must be a mode $n$ for which the spherical harmonic distortion has a maximum. This mode depends on $\Gamma$ as $n=\frac{1}{3} \sqrt{7+3 \Gamma}-\frac{2}{3}$. The number of fragments $N$ is estimated $N \approx n^{3}$.

Chomas et al. (2001b) showed that fragmentation occurs around peak contraction, when the bubble collapse is driven by inertial forces, since the inward acceleration continues to increase as the bubble approaches its minimum radius and suddenly changes sign as the bubble begins a rebound. They suggest that if the energy that is transferred during collapse is not sufficiently dissipated during one ultrasonic cycle, the bubble will become unstable and fragment.

Longuet-Higgins (1992) related the intrinsic energy of a bubble fragment population with a size distribution $P(R)$ to the initial intrinsic energy of the mother-bubble with equilibrium radius $R_{0}$ :

$$
E^{*}=\int_{0}^{\infty}\left(\frac{R}{R_{0}}\right)^{2} P(R) \mathrm{d} R,
$$

where $E^{*}$ is the intrinsic energy ratio, and $R$ is the radius of a fragment. For bubble fragments of equal sizes, $E^{*}=\frac{R_{0}}{R}=\sqrt[3]{N}$. Longuet-Higgins (1992) also presented an elegant method to compute the size distribution of bubbles generated by "shattering a single air cavity". 


\section{Sonic cracking}

Sonic cracking is the ultrasound-induced formation of a shell defect causing gas to escape from UCA microbubbles. It has been observed with rigid-shelled UCA microbubbles (Dayton et al., 1997a; Takeuchi, 1999; Postema et al., 2002a). The physical mechanism behind sonic cracking is yet not known. Tiny flaws in the shells may account for the fact that certain bubbles crack while others stay intact (Postema et al., 2002a).

\section{Jetting}

When a bubble rapidly contracts (collapses) near a boundary, this collapse will be asymmetrical. A high-speed liquid jet may form which projects through the bubble towards the boundary (Barnett, 1998), pulling a slight volume of the bubble content along. This remarkable phenomenon is called jetting.

The jetting phenomenon for cavitation bubbles was described by Philipp and Lauterborn (1998) and depicted by Ory ( $c f$. Figure 1): The asymmetric collapse causes the velocity of the upper bubble wall to exceed the velocity of the lower wall in order to conserve the impulse of the bubble/fluid system. Consequently, the fluid volume above the bubble is accelerated and focused during collapse, leading to the formation of a liquid jet directed towards the boundary. This jet hits the lower bubble wall, causing a funnel-shaped protrusion and finally impacts on 
the boundary.

Jets have regularly been observed with cavitation bubbles (Lauterborn, 1976; Philipp and Lauterborn, 1998). An illustrative enlargement of a high-speed photograph of a cavitation jet in the millimeter range was published by Lauterborn and Ohl (1997). Ohl and Ory (2000) calculated the shapes of asymmetrically collapsing bubbles. Their results are consistent with photographs of a collapsing cavitation bubble developing a jet. Kodama and Takayama (1998) studied the impact of jets on gelatin surfaces. They found that the radius of the jet $R_{\mathrm{j}}$ is approximately related to the radius of the bubble on the verge of collapsing $R_{\mathrm{c}}$ as $\frac{R_{\mathrm{j}}}{R_{\mathrm{c}}} \approx 0.1$ (Kodama and Takayama, 1998; Ohl and Ikink, 2003). The length of the jet $l_{\mathrm{j}}$, defined as the full travel path of the propelled liquid, is approximately related to $R_{\mathrm{c}}$ as $\frac{l_{\mathrm{j}}}{R_{\mathrm{c}}} \approx 3(\mathrm{Ohl}$ and Ikink, 2003). From these two ratios the amount of liquid within the jet, $V_{\mathrm{j}}$, is estimated $V_{\mathrm{j}} \approx 0.1 R_{\mathrm{c}}^{3}$ (Ohl and Ikink, 2003).

The impact of a jet on a surface generates a high-pressure region. The pressure in this region was dubbed water-hammer pressure by Cook (1928). For a perfectly plastic impact, the water-hammer pressure of a cavitation jet was estimated by de Haller (1933) and Ohl and Ory (2000):

$$
p_{\mathrm{wh}} \approx \frac{1}{2} \rho c v_{\mathrm{j}}
$$

where $p_{\mathrm{wh}}$ is the water-hammer pressure, $v_{\mathrm{j}}$ is the jet velocity, $\rho$ is the fluid density, and $c$ is the speed of sound. 
The damage caused by a jet can be estimated by comparing the water-hammer pressure to the maximum stress the impact surface can withstand before rupture. The maximum stress $\tau_{\max }$ is given by:

$$
\tau_{\max }=E \varepsilon_{\mathrm{r}}
$$

where $E$ is the elastic (Young's) modulus of the material, and $\varepsilon_{\mathrm{r}}$ is the relative deformation of the surface before rupture.

\section{Clinical applications}

UCA microbubble oscillating behavior is the basis of most diagnostic imaging techniques (Becher and Burns, 2000; Goldberg et al., 2001). Since oscillating microbubbles provide a means to discriminate blood from tissue, they are applicable in perfusion and flow imaging, and tumor detection (Burns et al., 1990; Wei et al., 1998; Wei, 2001).

Medical applications of the manipulation of the movement of UCA microbubbles by means of acoustic energy will be primarily in directing UCA toward cells (Dayton et al., 2001a).

If small UCA microbubbles, having passed through the narrowest vessels, coalesce, they may be controlled to obtain resonant sizes. Especially for subharmonic imaging (Shankar et al., 1999), where twice the resonant bubble size is needed, and for tracking the diffusion of free gas bubbles with subharmonics, a 
promising technique in noninvasive blood pressure measurements (Postema et al., 2003b), controlled microbubble coalescence may be applicable. If coalescence of a lipid-shelled microbubble and a cell membrane can be induced, this will imply a promising technique in targeted drug delivery.

Bouncing behavior of UCA microbubbles has got no potential medical application yet.

UCA microbubble fragmentation has been associated with violent effects, such as cell membrane permeabilization and lysis. The reason for this may lie in the fact that fragmenting bubbles produce shock-waves. Because of the strong acoustic signal generated, fragmentation finds applications in high-MI imaging. The applicability of microbubble fragmentation for local drug delivery is fully dependent of the presence of a shell after fragmentation. If the shell is absent after fragmentation, the fragments will behave as released gas bubbles. Thus, the potential therapeutic applications will then be identical to those for sonic cracking.

Sonic cracking may find applications in drug delivery, if the released bubble content has therapeutic properties (Frinking et al., 1998). Frinking et al. (2001) suggested a technique they called release burst imaging: Upon transmission of a high-MI ultrasound burst, there will be a strong scattering response from the released gas bubbles. The free gas will rapidly dissolve, and with it the strong scattering response will fade away. Wei et al. (1998) proposed that the acoustical 
measurement of the reappearance of UCA microbubbles may provide a means for determining tissue perfusion in any organ accessible to ultrasound.

The diffusion rate of a gas bubble with a certain size is known for every hydrostatic pressure. By making use of the changing acoustic properties of the dissolving gas bubbles, hydrostatic pressures may be determined in a minimally invasive clinical setting (Bouakaz et al., 2000; Postema et al., 2003b).

It has been speculated that jets may be formed when UCA microbubbles in the bloodstream are insonified with high enough acoustic pressures. These jets would be directed towards a boundary like a cell or a capillary wall with adjacent cells. As such, jets might function as microsyringes (Miller, 2000; Ohl and Ikink, 2003), if they could penetrate cells. The maximum extension measured in human cells is less than 50\% $\left(\varepsilon_{\mathrm{r}}<0.5\right)$ (Lendon et al., 1991). Therefore, we may assume that for any human cell or cell part, $\varepsilon_{\mathrm{r}} \ll 1$. Elastic moduli of human cells were measured $E \lesssim 7.3 \mathrm{kPa}$ for endothelial cells (Mathur et al., 2000), and $E \lesssim 12 \mathrm{kPa}$ for fibroblasts (Rotsch et al., 1999): less than half the elastic modulus of murine skeletal muscle cells ( $E \approx 24 \mathrm{kPa}$ ) (Mathur et al., 2001). Rabbit cardiac cells are among the stiffest, with $E \approx 100 \mathrm{kPa}$ (Mathur et al., 2001). It is safe to assume that even for the stiffest human cell $E \ll 200 \mathrm{kPa}$. Combining both assumptions in eq. (9) gives $\tau_{\max } \ll 200 \mathrm{kPa}$. If the water-hammer pressure of a jet exceeds this stress, the jet will be able to penetrate any human cell.

The porosities observed on cell-membranes by Tachibana et al. (1999) 
may well be attributed to jets. Probably, particles that were present in the photosensitive drug acted as cavitation nuclei, whereas the cell-membranes acted as the boundaries towards which the jets were directed.

\section{Experimental setup}

\section{Overview}

For the high-speed observations, we made use of the Brandaris-128 system (Chin et al., 2003) and an Imacon 468 fast framing camera. An overview of this experimental setup is shown in Figure 2. A computer controlled the triggering of a waveform generator, a Xenon flash source, and the cameras.

The electrical signal was generated by an AWG 520 arbitrary waveform generator (Tektronix, Inc., Beaverton, OR), or by an LW 420A arbitrary waveform generator (LeCroy Corp., Chestnut Ridge, NY). The signal was adjusted by two variable 355C/D attenuators (Hewlett Packard Company, Palo Alto, CA) in series, and an A-500 $60 \mathrm{~dB}$ linear power amplifier (ENI technology, Inc., Rochester, NY). It was converted to ultrasound by a v389-SU $500 \mathrm{kHz}$, or by a v397-SU 2.25 MHz single-element transducer (Panametrics Inc., Waltham, MA), both spherically focused at $7.5 \mathrm{~cm}$. The transducers were mounted in a Perspex container at an angle of $45^{\circ}$ relative to the top of the container. This container

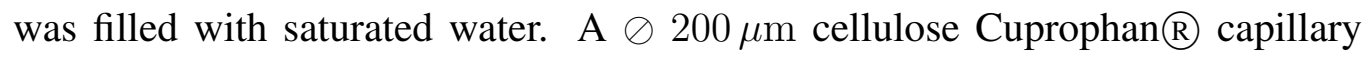


tube (Membrana $\mathrm{GmbH}$, Wuppertal, Germany) was fixed in the focal area of the transducer, through which contrast agent was flowing. Because the capillary tube was watersoaked and much smaller in diameter than the acoustic wavelenght, it was not expected to interfere with the ultrasound transmitted. Without contrast agent inserted, we did not observe reflections from the tube.

\section{Optics}

Underneath the capillary tube an optic fiber was mounted. This fiber was connected to an MVS-7010 Fiber Optic Strobe (PerkinElmer Optoelectronics, Salem, MA), and to a KLS-201 continuous fiber light source (Olympus KMI (KeyMed Ltd), Southend-on-Sea, UK). The pulsed light source was triggered by a PM 5716 pulse/delay generator (Koninklijke Philips Electronics N.V., Eindhoven, The Netherlands). The container was positioned beneath a customized BXFM microscope system (Olympus Optical Co., Ltd., Tokyo, Japan) with a U-CA magnification changer (Olympus Optical Co., Ltd.), switched to $2 \times$ magnification and a LUMPlanFl $60 \times$ water immersion objective lens (Olympus Optical Co., Ltd.). For the measurements with the Imacon 468 camera, we made use of a BH-2 model (Olympus Optical Co., Ltd.) with an SPlan 100 oil immersion objective lens (Olympus Optical Co., Ltd.). For image control purposes, an LCL-902K or a WAT-902HS CCD camera (Watec Co., Ltd., Yamagata, Japan) was fitted to the microscope. Focusing was done manually at the middle of the cellulose tube. 
Because the tube was wide compared to the contrast microbubbles measured, its upper half was considered a flat surface between contrast microbubbles and object lens, not causing aberrations but lowering the dynamic range of the images.

\section{Camera}

The optical observations were recorded with a Brandaris-128 fast framing camera system Chin et al. (2003). The Brandaris-128 captured sequences of 128 image frames at an average speed of 13 million frames per second. Typical frame sizes correspond to $89 \times 68 \mu \mathrm{m}^{2}$. In all observations, image frames were captured before, during, and after ultrasound insonification. A line drawing of the optical observation part of the setup is shown in Figure 3 and 4.

Furthermore, we made use of an Imacon 468 fast framing camera (DRS Hadland, Ltd., Tring, UK), capturing eight two-dimensional frames at $3 \mathrm{MHz}$. In all observations, the first frame was taken a few microseconds before ultrasound waves reached the contrast agent. The other seven frames were taken during ultrasound insonification, with $330 \mathrm{~ns}$ interframe time for $500 \mathrm{kHz}$ ultrasound, spanning a full ultrasound cycle. Frame exposure times ranged from $10 \mathrm{~ns}$ to $70 \mathrm{~ns}$. A line drawing of the optical observation part of this setup is shown in Figure 5. 


\section{Contrast agents}

We investigated the ultrasound contrast agent Quantison ${ }^{\mathrm{TM}}$ (Upperton Limited). It consists of human serum albumin-encapsulated air bubbles with a mean diameter of $3.2 \mu \mathrm{m}$. Shell thicknesses are between 0.2 and $0.3 \mu \mathrm{m}$ (Frinking and de Jong, 1998). The content of a Quantison ${ }^{\mathrm{TM}}$ vial was resuspended in $5 \mathrm{ml}$ of Isoton $\mathrm{R}$ II (Beckman Coulter, Inc., Fullerton, CA), and shaken gently for 20 seconds before further dilution. The agent was inserted through the capillary tube using a syringe pressed by hand.

We also investigated an experimental UCA (supplied by Bracco Research SA, Geneva, Switzerland). It consists of phospholipid-encapsulated gas bubbles ranging in diameter from 1 to $6 \mu \mathrm{m}$ with a median of $2 \mu \mathrm{m}$. The acoustic behavior of a very similar contrast agent was modeled and described in Gorce et al. (2000). Undiluted UCA ( $5 \mathrm{ml}$ of a $0.9 \%$ sodium chloride dilution, added to a $25 \mathrm{mg}$ UCA vial) was inserted through the capillary tube using either a syringe pressed by hand or a hose operated by a gravity fed or pumped infusion.

\section{Ultrasound}

For the experiments with the Brandaris- 128 camera system, the UCA bubbles were insonified by 8 cycles of $1.7 \mathrm{MHz}$ ultrasound at a peak rarefactional acoustic pressure of $2 \mathrm{MPa}$, corresponding to a mechanical index of $\mathrm{MI}=1.5$. For 
the experiments with the Imacon 468 camera, the UCA bubbles were insonified by 10 cycles of $500 \mathrm{kHz}$ ultrasound at peak rarefactional acoustic pressures between 0.66 and $0.85 \mathrm{MPa}$, corresponding to mechanical indices in the range $0.9<\mathrm{MI}<1.2$. The mechanical index is defined as MI $=p_{\text {ac }} / \sqrt{f}$, where $p_{\text {ac }}$ is the peak rarefactional acoustic pressure in MPa and $f$ is the center frequency of the ultrasound in $\mathrm{MHz}$.

Acoustic pressures applied were measured with a calibrated MH28-10 hydrophone (FORCE Technology, Brøndby, Denmark) in a separate water tank. An example of the acoustic signal measured at the transducer focus has been published in Postema et al. (2003a). The in-situ reverberant component due to the presence of the microscopic lens was observed to be less than $-14 \mathrm{~dB}$. Thus, it is assumed the in-situ acoustic signal is the same as the signal measured.

\section{Procedure}

We recorded 277 image sequences with the Brandaris-128 system. We performed 527 experiments at high acoustic amplitudes with the Imacond-468 camera. Bubble sizes and distances were measured manually or by using a segmentation method described by Postema et al. (2003a). 


\section{Results and discussion}

The following types of ultrasound-induced UCA microbubble behavior have been distinctly observed and categorized: oscillation, translation, coalescence, fragmentation, sonic cracking, and jetting.

\section{Oscillation}

Figure 6(i) shows an optical image sequence of two identical $\oslash 6 \mu \mathrm{m}$ moderately oscillating experimental UCA microbubbles freely flowing through a capillary tube. The frames have been taken during one cycle of ultrasound insonification, with a center frequency of $0.5 \mathrm{MHz}$ and a mechanical index $\mathrm{MI}=0.09$. Inter-frame times for frames $\mathrm{b}$ to $\mathrm{h}$ are $0.33 \mu \mathrm{s}$. Each frame corresponds to $\mathrm{a}$ $88 \times 58 \mu \mathrm{m}^{2}$ area. Frame a has been taken prior to ultrasound arrival. Maximal sizes are reached in frame $\mathrm{d}$ with bubble diameters of $7.6 \mu \mathrm{m}$, whereas minimal sizes are displayed in frame $\mathrm{f}$ with bubble diameters of $4.4 \mu \mathrm{m}$. Thus, the expansion and contraction of the bubbles are symmetric. We do not expect a violent collapse in this regime, since the maximum bubble radius is less than twice its equilibrium radius. The oscillating amplitude is represented in the left frame of Figure 6(iii). The solid line represents the radius-period curve of a free gas bubble (de Jong et al., 1994). Apparently, the UCA bubbles have comparable excursions to a free gas bubble. 
Figure 6(ii) and the right frame of Figure 6(iii) show a similar situation for a $\oslash 1.5 \mu \mathrm{m}$ strongly oscillating microbubble insonified at $\mathrm{MI}=0.67$. Again, the UCA bubble excursion is comparable to a free gas bubble. At the acoustic amplitude applied there may be some energy loss in the shell, causing increased thermal damping. Maximum size is reached in frame e with a bubble diameter of $10.0 \mu \mathrm{m}$, whereas minimum size is displayed in frame $\mathrm{g}$ with a bubble diameter of $0.57 \mu \mathrm{m}$. The bubble oscillates highly asymmetric. We expect the microbubble to grow into an inertial cavity in this regime, since the maximum bubble radius is much greater than its equilibrium radius.

\section{Translation}

Figure 7 shows a high-speed optical image sequence of Quantison ${ }^{\mathrm{TM}}$ insonified by 8 cycles of $1.7 \mathrm{MHz}$ ultrasound with a mechanical index MI $=1.5$. The ultrasound travels from the lower side of the frames to the upper side. Frame times are indicated in ns. Each frame corresponds to a $30 \times 16 \mu \mathrm{m}^{2}$ area. In Figure 7(b,c,d) gas escapes from a $\oslash 4 \mu \mathrm{m}$ bubble. This free gas bubble expands and contracts, and translates towards the upper side of the frames. The displacement of the free gas bubble center has been measured $14 \mu \mathrm{m}$ over 4 periods (frames e-h). The resting diameter of the released bubble after insonification was $1.3 \mu \mathrm{m}$. The theoretical mean bubble displacement over time has been computed by integrating eq. (1) over 4 periods, taking $c=1480 \mathrm{~m} \mathrm{~s}^{-1}, \rho=998 \mathrm{~kg} \mathrm{~m}^{-3}, R_{0}=0.65 \mu \mathrm{m}$, 
$f=1.7 \mathrm{MHz}, f_{\mathrm{r}}=5.3 \mathrm{MHz}, \delta_{\mathrm{t}}=1.0, p_{\mathrm{ac}}(t)=[2 \mathrm{MPa}] \sin 2 \pi f t$, and $\eta=0.001 \mathrm{Pas}$. The damping was computed using the parameters stated in Medwin (1977) and de Jong (1993). The theoretical displacement is $18 \mu \mathrm{m}$. The measured displacement is lower than the theoretical value. At the acoustic pressure applied, the expansion phase is longer than the contraction phase, which may account for this difference.

Figure 8 shows 5 image frames of two $\oslash 4 \mu \mathrm{m}$ experimental UCA microbubbles, each captured after insonification by 10 cycles of $0.5 \mathrm{MHz}$ ultrasound (duration $T_{\mathrm{p}}=20 \mu \mathrm{s}$ ). Each image frame corresponds to a $30 \times 20 \mu \mathrm{m}^{2}$ area. During every ultrasound burst the bubbles draw nearer to each other. For each center-to-center distance $d_{0}$ measured the mean approach velocity $v_{\mathrm{a}}$ has been computed from eq. (4), taking $\kappa=5 \times 10^{-6} \mathrm{~m}^{2} \mathrm{~N}^{-1}$. By combining $d_{0}$ with $v_{\mathrm{a}}$, the theoretical distances $\Delta d_{\mathrm{th}}=v_{\mathrm{a}} T_{\mathrm{p}}$ have been computed. These were compared to the distance $\Delta d_{\mathrm{m}}$ measured from Figure 8 . The results are summarized in Table 1 . The measured values $\Delta d$ are consistent with theory.

\section{Coalescence}

Figure 9(i) shows an example of microbubble coalescence. The first frame has been captured prior to ultrasound arrival. In Figure 9(i)b), the main bubble with an initial diameter of $4 \mu \mathrm{m}$ has split up, into several fragments with diameters below or around optical resolution, and these fragments have started to coalesce 
upon expansion. As the resulting two bubbles have expanded to mean diameters of approximately $2.7 \mu \mathrm{m}$, shown in Figure 9(i)c), the flattening of the adjacent bubble surfaces is clearly visible. After the bubbles have expanded further to mean diameters of $4.6 \mu \mathrm{m}$, as demonstrated in Figure 9(i)d, the interposed film has drained to a critical thickness to rupture (Figure 9(i)e), which has resulted in the formation of a single bubble (Figure 9(i)f).

The flattening of the adjacent bubble surfaces in Figure 9(i)b) is supported by a conservative estimate of the Weber number. Based on our analysis of the optical system (Postema et al., 2003a), we may assume that the optical resolution $\mathcal{R} \ll 0.5 \mu \mathrm{m}$, and fragment diameters in Figure 9(i)b) $<0.5 \mu \mathrm{m}$. Even if the bubbles would expand linearly to the sizes observed in Figure 9(i)c, the approach velocity would still have to be $u=2 \frac{\Delta R_{\mathrm{m}}}{\Delta t}>\frac{2.7 \mu \mathrm{m}-0.5 \mu \mathrm{m}}{0.33 \mu \mathrm{s}}=6.7 \mathrm{~ms}^{-1}$. By taking $\rho=998 \mathrm{~kg} \mathrm{~m}^{3}$ and $\sigma=0.072 \mathrm{~N} \mathrm{~m}^{-1}$, we obtain a Weber number We $>0.84$. As stated before, flattening of the adjacent bubble surfaces will occur if We $>0.5$.

From eq. (6), it is expected that the drainage time of the film shown in Figure 9(i)c until a critical thickness $h_{\mathrm{c}}=0.1 \mu \mathrm{m}$ is $t_{\mathrm{d}}=0.6 \mu \mathrm{m}$, taking $\eta=0.001 \mathrm{Pas}$ and $R_{\mathrm{f}} \approx \frac{2}{3} R_{\mathrm{m}}$ (Postema et al., 2003c). However, Figure 9(i)d shows not only film drainage, but also continuing bubble expansion. For these bubble diameters of $4.6 \mu \mathrm{m}$, the interposed film would take $2.8 \mu$ s to drain. Still, $0.66 \mu$ s later the bubbles have coalesced, although the bubbles have continued to expand. This supports the theory that a mechanism for film rupture must be at 
work that overrules the drainage.

\section{Fragmentation}

Figure 9(ii) shows the repeated fragmentation, coalescence, and re-fragmentation of an experimental UCA microbubble with an initial diameter $\mathcal{D}=6 \mu \mathrm{m}$. Each frame corresponds to a $23 \times 23 \mu \mathrm{m}^{2}$ area. Figure 9(ii)a was captured prior to ultrasound arrival. In Figure 9(ii)b the bubble has broken up into 8 discernable fragments, suggesting a mode $n=\sqrt[3]{8}=2$ spherical harmonical instability. The fragments coalesce and form an ellipsoidal bubble in Figure 9(ii)e. Because the ellipsoidal shape can be discriminated, we may conclude that the second spherical harmonic mode is dominant. After collapse, the fragments in Figure 9(ii)f are too small to be counted, but in Figure 9(ii)g, captured while the fragments have begun to expand, 8 fragments can be discerned, which confirms the second mode instability.

\section{Sonic cracking}

Figure 9(iii) shows the sonic cracking of a $\oslash 3 \mu \mathrm{m}$ Quantison $^{\mathrm{TM}}$ microbubble. Each frame corresponds to a $23 \times 23 \mu \mathrm{m}^{2}$ area. In Figure 9(iii)d gas starts to escape from the bubble in the middle of the frame. It expands to approximately $\oslash 8 \mu \mathrm{m}$ in Figure 9(iii)g, and then begins to contract in Figure 9(iii)h.

Clearly, the gas bubble has been released from its rigid shell. However, in the 
image sequence, the pixel size $\left(0.15 \times 0.15 \mu \mathrm{m}^{2}\right)$ corresponds in approximation to the shell thickness. Thus, even if the optical resolution were of the order of the shortest wavelength of the light, the presence of tiny flaws in the rigid shell would not have been detectable.

\section{Jetting}

We recorded two optical sequences showing jet development in UCA microbubbles (Postema et al., 2002b), one of which is shown in Figure 10. Figure 10(a) shows a microbubble with a radius $R_{\mathrm{c}}=8.43 \mu \mathrm{m}$. Figure $10(\mathrm{~b})$ has been captured $0.33 \mu \mathrm{s}$ later. Liquid is propelled through the lower left and escapes from the upper right of the bubble. The jet has traveled over a length $l_{\mathrm{j}}=26.2 \mu \mathrm{m}$ in $0.33 \mu \mathrm{s}$, giving an average jet velocity $v_{\mathrm{j}}=79.4 \mathrm{~m} \mathrm{~s}^{-1}$. The ratio $\frac{l_{\mathrm{j}}}{R_{\mathrm{c}}}$ is in agreement with the ratio put forward by Ohl and Ikink (2003). The volume of the jet is approximately $V_{\mathrm{j}} \approx 0.1 R_{\mathrm{c}}^{3}=60$ femtoliter.

Taking $\rho=998 \mathrm{~kg} \mathrm{~m}^{-3}$ and $c=1480 \mathrm{~m} \mathrm{~s}^{-2}$, the water-hammer pressure is around $60 \mathrm{MPa}$. Since $p_{\mathrm{wh}} \gg 200 \mathrm{kPa} \gg \tau_{\max }$, such a jet may penetrate any cell.

\section{Influence of the capillary tube}

Ishida et al. (2001) demonstrated with simulations and high-speed optical observations, that if the distance between the walls of their solid test vessel was 
greater than 5 times the maximum free-field expansion radius of a cavitation bubble, the bubble would remain spherical while expanding. In our setup, the diameter of the water-soaked capillary tube is approximately 20 times the largest microbubble radius measured. Thus, the phenomena described other than jetting cannot be attributed to the boundaries imposed by the capillary tube, since our imaging plane is at the center of the tube.

\section{Conclusions}

An overview of dynamic behavior of ultrasound insonified encapsulated microbubbles has been presented. The following types of behavior have been observed and categorized: oscillation, translation, coalescence $\&$ bounce, fragmentation, sonic cracking, and jetting.

Oscillation is the expanding and contracting bubble response to an oscillating acoustic signal. At low acoustic amplitudes bubbles pulsate moderately, at high amplitudes their longer expansion phase is followed by a violent collapse. Microbubble translation has been associated with primary and secondary radiation forces. Our optical observations of translating bubbles are consistent with theory. Coalescence, the fusion of two or more bubbles, is mainly caused by the drainage of the liquid film separating expanding bubbles, whereas bounce - unsuccessful coalescence - is caused by the drainage taking longer than the expansion of 
the bubbles. Fragmentation is the fission of a bubble into smaller bubbles. The number of fragments has been related to the dominant spherical harmonical oscillation mode of a bubble. In our observations, the second spherical harmonical mode appears to be the dominant mode. Remarkable are our observations of jetting through encapsulated microbubbles. For an observed jet, we computed a volume of approximately 60 femtoliter, generating a pressure at the tip of the jet around $60 \mathrm{MPa}$. This is high enough to penetrate any human cell. Hence, liquid jets may act as microsyringes, delivering a drug to a region of interest.

Table 2 gives an overview of potential clinical applications related to the encapsulated microbubble phenomena discussed. The phenomena have potential clinical applications in imaging, pressure measurements, tumor detection, permeabilization, lysis, targeting, and drug delivery.

\section{Acknowledgments}

This work has been supported by the Technology Foundation STW (RKG.5104). We thank Kees Slager for the data on cell elasticity, Emmanuel Ory (Laboratory of Applied Thermodynamics, Helsinki University of Technology) for computing Figure 1, Leo Bekkering and Elmer Koene for technical assistance, and Cees Pakvis for drawing Figures 3, 4, and 5. 


\section{References}

Barnett S. Nonthermal issues: cavitation - its nature, detection and measurement. Ultrasound Med Biol 1998;24:S11-S21.

Becher H, Burns PN. Handbook of Contrast Echocardiography: LV Function and Myocardial Perfusion. Berlin: Springer-Verlag 2000.

Bouakaz A, Frigstad S, Ten Cate FJ, de Jong N. Improved contrast to tissue ratio at higher harmonics. Ultrasonics 2002a;40:575-578.

Bouakaz A, Frigstad S, Ten Cate FJ, de Jong N. Super harmonic imaging: a new imaging technique for improved contrast detection. Ultrasound Med Biol 2002b;28(1):59-68.

Bouakaz A, Frinking PJA, de Jong N. Noninvasive pressure measurement using microbubble contrast agent and wavelet transforms. Proc IEEE Ultrason Symp 2000;1907-1910.

Brennen CE. Fission of collapsing cavitation bubbles. Proc CAV 2001 4th Int Symp Cavitation 2001;

Burns PN, Hilpert P, Goldberg BB. Intravenous contrast agent for ultrasound Doppler: In vivo measurement of small tumor vessel dose-response. Proc Annu Int Conf IEEE Eng Med Biol Soc 1990;12(1):322-324. 
Chesters AK, Hoffmann G. Bubble coalescence in pure liquids. Appl Sci Res $1982 ; 38: 353-361$.

Chin CT, Lancée C, Borsboom J, Mastik F, Frijlink M, de Jong N. Brandaris 128: a 25 million frames per second digital camera with 128 highly sensitive frames. Rev Sci Instru 2003;74(12):5026-5034.

Chiou KR, Liu CP, Chiang HT, Lin SL. Clinical applications of contrast echocardiography. Acta Cardiol Sin 2000;16:63-78.

Chomas JE, Dayton P, Allen J, Morgan K, Ferrara KW. Mechanisms of contrast agent destruction. IEEE Trans Ultrason, Ferroelect, Freq Contr 2001a; 48(1):232-248.

Chomas JE, Dayton P, May D, Ferrara K. Threshold of fragmentation for ultrasonic contrast. J Biomed Opt 2001b;6(2):141-150.

Cook SS. Erosion by water-hammer. Proc Roy Soc London A 1928;119:481-488.

Dayton P, Goode A, Morgan K, Klibanov S, Brandenburger G, Ferrara K. Action of microbubbles when insonified: experimental evidence. Proc IEEE Ultrason Symp 1996;1131-1134.

Dayton P, Klibanov A, Brandenburger G, Ferrara K. Acoustic radiation force in vivo: a mechanism to assist targeting of microbubbles. Ultrasound Med Biol 1999;25(8):1195-1201. 
Dayton P, Morgan K, Allietta M, Klibanov A, Brandenburger G, Ferrara K. Simultaneous optical and acoustical observations of contrast agents. Proc IEEE Ultrason Symp 1997a;1583-1591.

Dayton PA, Allen JS, Kruse DE, Ferrara KW. Experimental validation of a theoretical framework to predict radiation force displacement of contrast agents. Proc IEEE Ultrason Symp 2001a;1687-1690.

Dayton PA, Chomas JE, Lum AFH, Allen JS, Lindner JR, Simon SI, Ferrara KW. Optical and acoustical dynamics of microbubble contrast agents inside neutrophils. Biophys J 2001b;80:1547-1556.

Dayton PA, Morgan KE, Klibanov AL, Brandenburger G, Nightingale KR, Ferrara KW. A preliminary evaluation of the effects of primary and secondary radiation forces on acoustic contrast agents. IEEE Trans Ultrason, Ferroelect, Freq Contr 1997b;44(6):1264-1277.

de Haller P. Untersuchungen über die durch Kavitation hergerufenen Korrosionen. Schweiz Bauzeit 1933;101(21):243-246.

de Jong N. Acoustic properties of ultrasound contrast agents. Rotterdam: N. de Jong 1993.

de Jong N, Bouakaz A, Ten Cate FJ. Contrast harmonic imaging. Ultrasonics 2002;40:567-573. 
de Jong N, Cornet R, Lancée CT. Higher harmonics of vibrating gas-filled microspheres. Part one: simulations. Ultrasonics 1994;32(6):447-453.

de Jong N, Frinking PJA, Bouakaz A, Goorden M, Schuurmans T, Jingping X, Mastik F. Optical imaging of contrast agent microbubbles in an ultrasound field with a 100-MHz camera. Ultrasound Med Biol 2000a;26(3):487-492.

de Jong N, Frinking PJA, Bouakaz A, Ten Cate FJ. Detection procedures of ultrasound contrast agents. Ultrasonics 2000b;38:87-92.

Duineveld PC. Bouncing and coalescence of two bubbles in water. Enschede: P.C. Duineveld 1994.

Flynn HG. Cavitation dynamics. II. Free pulsations and models for cavitation bubbles. J Acoust Soc Am 1975;58(6):1160-1170.

Flynn HG, Church CC. Transient pulsations of small gas bubbles in water. J Acoust Soc Am 1988;84(3):985-998.

Frinking PJA, Bouakaz A, de Jong N, Ten Cate FJ, Keating S. Effect of ultrasound on the release of micro-encapsulated drugs. Ultrasonics 1998;36:709-712.

Frinking PJA, Céspedes EI, Kirkhorn J, Torp HG, de Jong N. A new ultrasound contrast imaging approach based on the combination of multiple imaging pulses and a separate release burst. IEEE Trans Ultrason, Ferroelect, Freq Contr 2001; 48(3):643-651. 
Frinking PJA, de Jong N. Acoustic modeling of shell-encapsulated gas bubbles. Ultrasound Med Biol 1998;24(4):523-533.

Goldberg BB, Raichlen JS, Forsberg F, eds. Ultrasound Contrast Agents. Basic principles and clinical applications. London: Martin Dunitz Ltd, 2nd edition 2001.

Gorce JM, Arditi M, Schneider M. Influence of bubble size distribution on the echogenicity of ultrasound contrast agents: A study of SonoVue ${ }^{\mathrm{TM}}$. Invest Radiol 2000;35(11):661-671.

Hilgenfeldt S, Brenner MP, Grossmann S, Lohse D. Analysis of Rayleigh-Plesset dynamics for sonoluminescing bubbles. J Fluid Mech 1998;365:171-204.

Ishida H, Nuntadusit C, Kimoto H, Nakagawa T, Yamamoto T. Cavitation behavior near solid boundaries. Proc CAV 2001 4th Int Symp Cavitation 2001;

Klibanov AL. Targeted delivery of gas-filled microspheres, contrast agents for ultrasound imaging. Adv Drug Delivery Rev 1999;37:139-157.

Kodama T, Takayama K. Dynamic behavior of bubbles during extracorporeal shock-wave lithotripsy. Ultrasound Med Biol 1998;24(5):723-738.

Kralchevsky PA, Danov KD, Ivanov IB. Thin liquid film physics. In: 
R Prud'homme, S Khan, eds., Foams, Theory, Measurements and Applications, New York: Marcel Dekker 1996; 1-98.

Kudo N, Miyaoka T, Okada K, Yamamoto K, Niwa K. Study on mechanism of cell damage caused by microbubbles exposed to ultrasound. Proc IEEE Ultrason Symp 2002;1351-1354.

Lauterborn W. Optische Kavitation. Physikalische Blätter 1976;32(12):553-563.

Lauterborn W, Ohl CD. Cavitation bubble dynamics. Ultrason Sonochem 1997; $4: 65-75$.

Leighton TG. The Acoustic Bubble. London: Academic Press Ltd. 1994.

Lendon CL, Davies MJ, Born GVR, Richardson PD. Atherosclerotic plaque caps are locally weakened when macrophages density is increased. Atherosclerosis 1991;87:87-90.

Longuet-Higgins MS. The crushing of air cavities in a liquid. Proc Roy Soc London A 1992;439:611-626.

Marmottant P, Hilgenfeldt S. Controlled vesicle deformation and lysis by single oscillating bubbles. Nature 2003;423:153-156.

Maruvada S, Hynynen K. Optical monitoring of ultrasound interaction with blood 
vessels in transparent fish after injection with contrast agents. Proc IEEE Ultrason Symp 2002;1936-1939.

Mathur AB, Collinsworth AM, Reichert WM, Kraus WE, Truskey GA. Endothelial, cardiac muscle and skeletal muscle exhibit different viscous and elastic properties as determined by atomic force microscopy. J Biomech 2001; $34: 1545-1553$.

Mathur AB, Truskey GA, Reichert WM. Atomic force and total internal reflection fluorescence microscopy for the study of force transmission in endothelial cells. Biophys J 2000;78:1725-1735.

Medwin H. Counting bubbles acoustically: a review. Ultrasonics 1977;15:7-13.

Miller DL, Nyborg WL. Theoretical investigation of the response of gas-filled micropores and cavitation nuclei to ultrasound. J Acoust Soc Am 1983; 73(5):1537-1544.

Miller MW. Gene transfection and drug delivery. Ultrasound Med Biol 2000; 26(S1):S59-S62.

Morgan KE, Allen JS, Dayton PA, Chomas JE, Klibanov AL, Ferrara KW. Experimental and theoretical evaluation of microbubble behavior: Effect of transmitted phase and bubble size. IEEE Trans Ultrason, Ferroelect, Freq Contr 2000;47(6):1494-1509. 
Narsimhan G, Ruckenstein E. Structure, drainage, and coalescence of foams and concentrated emulsions. In: R Prud'homme, S Khan, eds., Foams, Theory, Measurements and Applications, New York: Marcel Dekker 1996; 99-187.

Ohl CD, Ikink R. Shock-wave-induced jetting of micron-size bubbles. Phys Rev Lett 2003;90:214502.

Ohl CD, Ory E. Aspherical bubble collapse - comparison with simulations. In: W Lauterborn, T Kurz, eds., Nonlinear Acoustics at the Turn of the Millennium: ISNA 15, American Institute of Physics 2000; 393-396.

Pain HJ. The physics of vibrations and waves. Chichester: John Wiley \& Sons Ltd, 3rd edition 1992.

Philipp A, Lauterborn W. Cavitation erosion by single laser-produced bubbles. J Fluid Mech 1998;361:75-116.

Postema M, Bouakaz A, Chin CT, de Jong N. Real-time optical imaging of individual microbubbles in an ultrasonic field. Proc IEEE Ultrason Symp 2001; $1679-1682$.

Postema M, Bouakaz A, Chin CT, de Jong N. Optically observed microbubble coalescence and collapse. Proc IEEE Ultrason Symp 2002a;1900-1903.

Postema M, Bouakaz A, Chin CT, de Jong N. Simulations and measurements 
of optical images of insonified ultrasound contrast microbubbles. IEEE Trans Ultrason, Ferroelect, Freq Contr 2003a;50(5):523-536.

Postema M, Bouakaz A, de Jong N. IEEE Trans Ultrason, Ferroelect, Freq Contr 2002b;49(3):cover page.

Postema M, Bouakaz A, de Jong N. Noninvasive microbubble-based pressure measurements: a simulation study. Ultrasonics 2003b;in press.

Postema M, Lancée CT, de Jong N, Marmottant P, Hilgenfeldt S. Ultrasound-induced microbubble coalescence 2003c;submitted for publication.

Reddy AJ, Szeri AJ. Coupled dynamics of translation and collapse of acoustically driven microbubbles. J Acoust Soc Am 2002;112(4):1346-1352.

Rotsch C, Jacobson K, Radmacher M. Dimensional and mechanical dynamics of active and stable edges in motile fibroblasts investigated by using atomic force microscopy. Proc Natl Acad Sci USA 1999;96:921-926.

Schrope BA, Newhouse VL. Second harmonic ultrasonic blood perfusion measurement. Ultrasound Med Biol 1993;19(7):567-579.

Shankar PM, Krishna PD, Newhouse VL. Subharmonic backscattering from ultrasound contrast agents. J Acoust Soc Am 1999;106(4):2104-2110.

Sheludko A. Thin liquid films. Advan Colloid Interf Sci 1967;1:391-464. 
Shi WT, Forsberg F, Tornes A, Østensen J, Goldberg BB. Destruction of contrast microbubbles and the association with inertial cavitation. Ultrasound Med Biol 2000;26(6):1009-1019.

Shohet RV, Chen S, Zhou YT, Wang Z, Meidell RS, Unger RH, Grayburn PA. Echocardiographic destruction of albumin microbubbles directs gene delivery to the myocardium. Circulation 2000;101:2554-2556.

Skyba DM, Price RJ, Linka AZ, Skalak TC, Kaul S. Direct in vivo visualization of intravascular destruction of microbubbles by ultrasound and its local effects on tissue. Circulation 1998;98:290-293.

Strasberg M. Gas bubbles as sources of sound in liquids. J Acoust Soc Am 1956; 28(1):20-26.

Stride E, Saffari N. On the destruction of microbubble ultrasound contrast agents. Ultrasound Med Biol 2003;29(4):563-573.

Tachibana K, Uchida T, Ogawa K, Yamashita N, Tamura K. Induction of cell-membrane porosity by ultrasound. Lancet 1999;353:1409.

Takeuchi Y. IEEE Trans Ultrason, Ferroelect, Freq Contr 1999;46(4):cover page.

Taniyama Y, Tachibana K, Hiraoka K, Namba T, Yamasaki K, Hashiya N, Aoki M, Ogihara T, Yasufumi K, Morishita R. Local delivery of plasmid DNA into rat carotid artery using ultrasound. Circulation 2002;105:1233-1239. 
Tortoli P, Michelassi V, Corsi M, Righi D, Takeuchi Y. On the interaction between ultrasound and contrast agents during Doppler investigations. Ultrasound Med Biol 2001;27(9):1265-1273.

Uhlendorf V, Hoffmann C. Nonlinear acoustical response of coated microbubbles in diagnostic ultrasound. Proc IEEE Ultrason Symp 1994;1559-1562.

Unger EC, Matsunaga TO, McCreery T, Schumann P, Sweitzer R, Quigley R. Therapeutic applications of microbubbles. Eur J Radiol 2002;42:160-168.

Unger EC, Wu Q, McGreery TP, Matsunaga TO. Thrombus-specific contrast agents for imaging and thrombolysis. In: B Goldberg, J Raichlen, F Forsberg, eds., Ultrasound Contrast Agents. Basic principles and clinical applications, London: Martin Dunitz Ltd. 2nd edition 2001; 337-345.

van Wamel A, Bouakaz A, ten Cate F, de Jong N. Effects of diagnostic ultrasound parameters on molecular uptake and cell viability. Proc IEEE Ultrason Symp $2002 ; 1387-1390$.

Vokurka K. Comparison of Rayleigh's, Herring's, and Gilmore's models of gas bubbles. Acustica 1986;59:214-219.

Wei K. Blood flow and perfusion measurements: background and clinical utility. In: B Goldberg, J Raichlen, F Forsberg, eds., Ultrasound Contrast Agents. Basic 
principles and clinical applications, London: Martin Dunitz Ltd. 2nd edition $2001 ; 89-104$.

Wei K, Jayaweera AR, Firoozan S, Linka A, Skyba DM, Kaul S. Quantification of myocardial blood flow with ultrasound-induced destruction of microbubbles administered as a constant venous infusion. Circulation 1998;97:473-483. 


\section{Tables}

Table 1: Traveled distances and mean velocities of approaching bubbles.

\begin{tabular}{ccccc}
\hline frame & $\begin{array}{c}d_{0} \\
(\mu \mathrm{m})\end{array}$ & $\begin{array}{c}v_{\mathrm{a}} \\
\left(\mathrm{cm} \mathrm{s}^{-1}\right)\end{array}$ & $\begin{array}{c}\Delta d_{\mathrm{th}} \\
(\mu \mathrm{m})\end{array}$ & $\begin{array}{c}\Delta d_{\mathrm{m}} \\
(\mu \mathrm{m})\end{array}$ \\
\hline \hline $\mathrm{a}$ & 21.2 & 15 & 3.0 & 3.2 \\
$\mathrm{~b}$ & 18.0 & 20 & 4.0 & 3.9 \\
$\mathrm{c}$ & 14.1 & 33 & 6.6 & 6.4 \\
$\mathrm{~d}$ & 7.7 & 111 & $>7.7$ & 7.7 \\
$\mathrm{e}$ & 0 & & & \\
\hline
\end{tabular}

Table 2: Potential clinical applications for encapsulated microbubble phenomena observed.

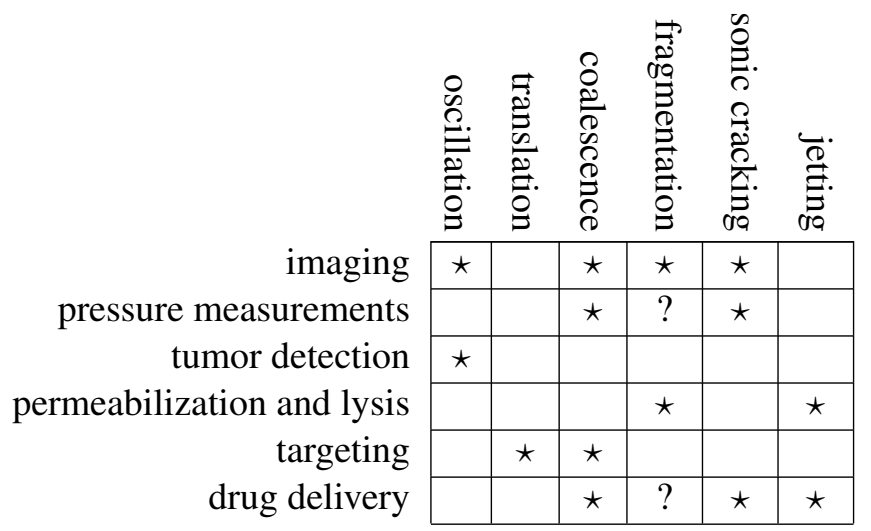




\section{List of Figures}

1 Schematic evolution of jet development in a collapsing bubble near a boundary (below): bubble on the verge of collapse (a), asymmetric collapse (b), jet development (c), funnel-shaped protrusion $(\mathrm{d}) . \ldots \ldots \ldots \ldots \ldots$. . . . . . . . . . . . . .

2 Basic setup of the high-speed photography system. . . . . . . 48

3 Line drawing of the Brandaris-128 system. . . . . . . . . . . . . 49

4 Line drawing of the optical observation part of the Brandaris-128 system. ....................... 50

5 Line drawing of the optical observation of the Imacon-468 system. 51

6 Oscillating experimental UCA microbubbles. Both respective image sequences span one ultrasonic cycle. Frames a have been captured prior to ultrasound arrival. Inter-frame times for frames $\mathrm{b}$ to $\mathrm{h}$ are $0.33 \mu \mathrm{s}$. Each frame corresponds to a $88 \times 58 \mu \mathrm{m}^{2}$ area. (i) Two $\oslash 6 \mu \mathrm{m}$ bubbles moderately oscillating (MI $=0.09$ ). (ii) $\mathrm{A} \oslash 1.5 \mu \mathrm{m}$ bubble strongly oscillating (MI $=0.67$ ). (iii) Radius-period plots of both events. The solid line represents an oscillating free gas bubble. . . . . . . . . . . . . 52 
$7 \quad$ (a-d) Sonic cracking of a $\oslash 4 \mu \mathrm{m}$ Quantison ${ }^{\mathrm{TM}}$ bubble $(\mathrm{MI}=1.5)$.

(e-i) The released gas is subjected to a primary radiation force. Each image frame corresponds to a $30 \times 16 \mu \mathrm{m}^{2}$ area. Frames shown were selected from a sequence of 128 frames. The travel distance of the bubble center between (e) and (h) is $14 \mu \mathrm{m}$ over 4 ultrasonic cycles. . . . . . . . . . . . . . 53

8 The approach of two $\oslash 4 \mu \mathrm{m}$ experimental UCA bubbles induced by a secondary radiation force. Each image frame corresponds to a $30 \times 20 \mu \mathrm{m}^{2}$ area. Frames are each captured after insonification by 10 cycles of $0.5 \mathrm{MHz}$ ultrasound $(\mathrm{MI}=0.67) . \ldots \ldots 54$

9 Experimental UCA microbubbles showing (i) coalescence, (ii) second mode spherical harmonical fragmentation, (iii) sonic cracking. Frames a have been captured prior to ultrasound arrival. Inter-frame times for frames $\mathrm{b}$ to $\mathrm{h}$ are $0.33 \mu \mathrm{s}$. Each image frame corresponds to a $23 \times 23 \mu \mathrm{m}^{2}$ area. . . . . . . . . . 55

10 Ultrasound-induced jet development in an experimental UCA microbubble $(\mathrm{MI}=1.2)$. Each image frame corresponds to a $38 \times 30 \mu \mathrm{m}^{2}$ area. Inter-frame time is $0.33 \mu \mathrm{s}$ and exposure time is $10 \mathrm{~ns} \ldots \ldots \ldots \ldots \ldots \ldots \ldots$ 


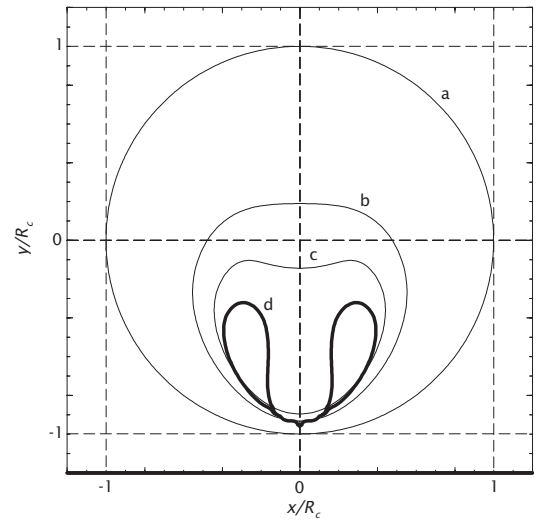

Figure 1: Schematic evolution of jet development in a collapsing bubble near a boundary (below): bubble on the verge of collapse (a), asymmetric collapse (b), jet development (c), funnel-shaped protrusion (d). 


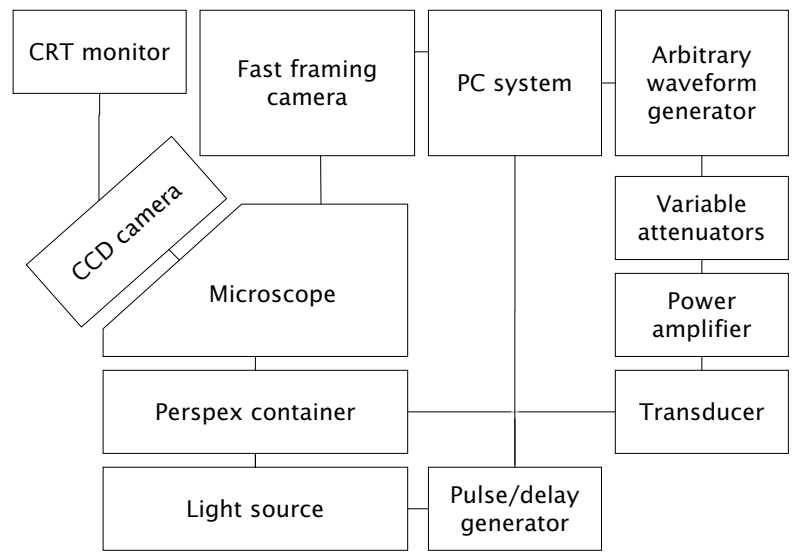

Figure 2: Basic setup of the high-speed photography system. 


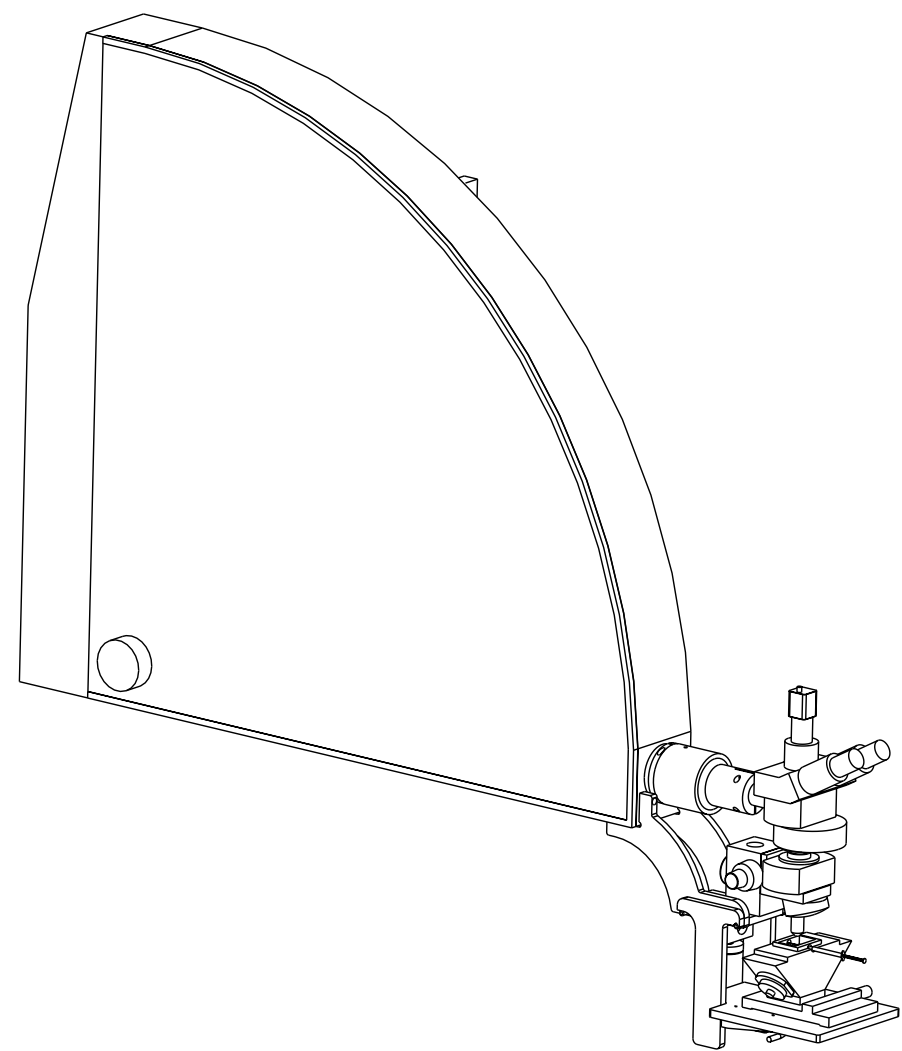

Figure 3: Line drawing of the Brandaris-128 system. 


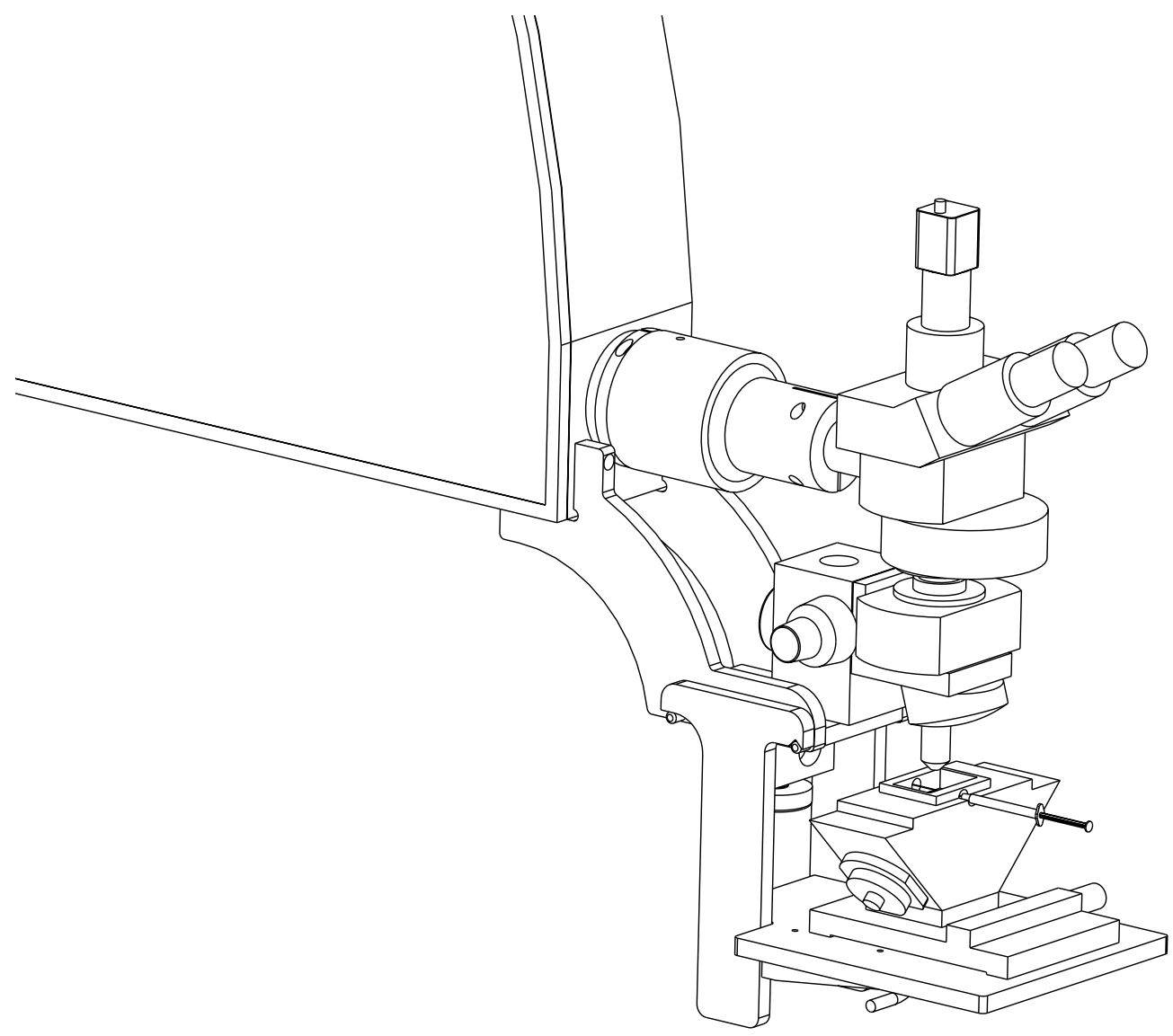

Figure 4: Line drawing of the optical observation part of the Brandaris-128 system. 


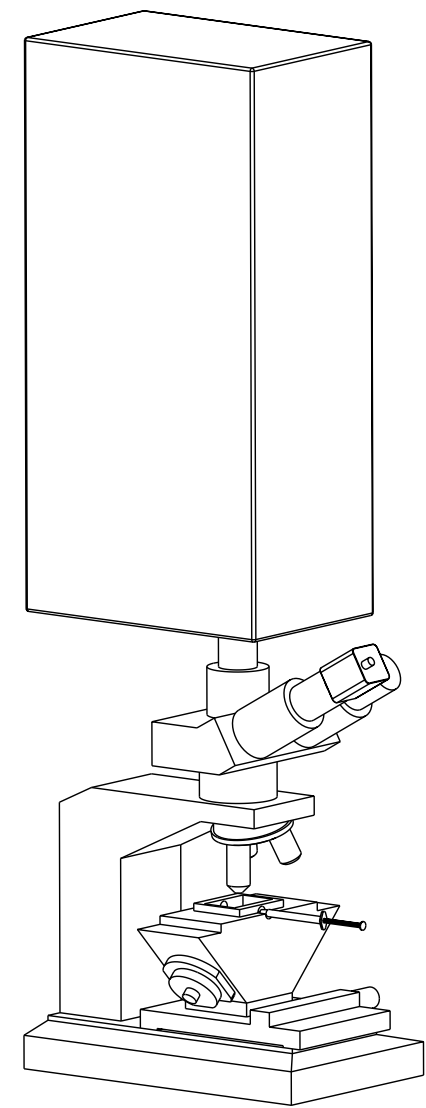

Figure 5: Line drawing of the optical observation of the Imacon-468 system. 


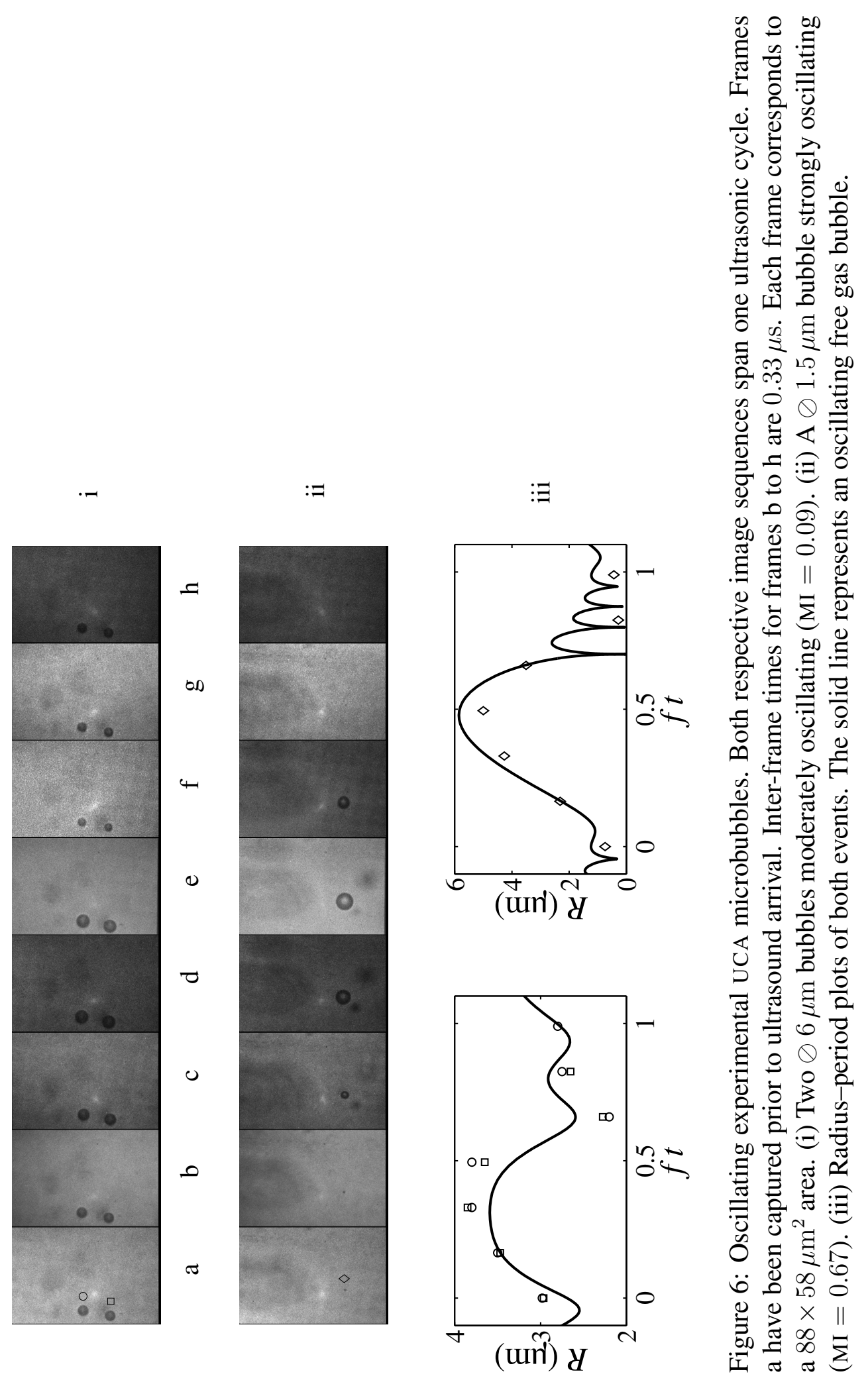




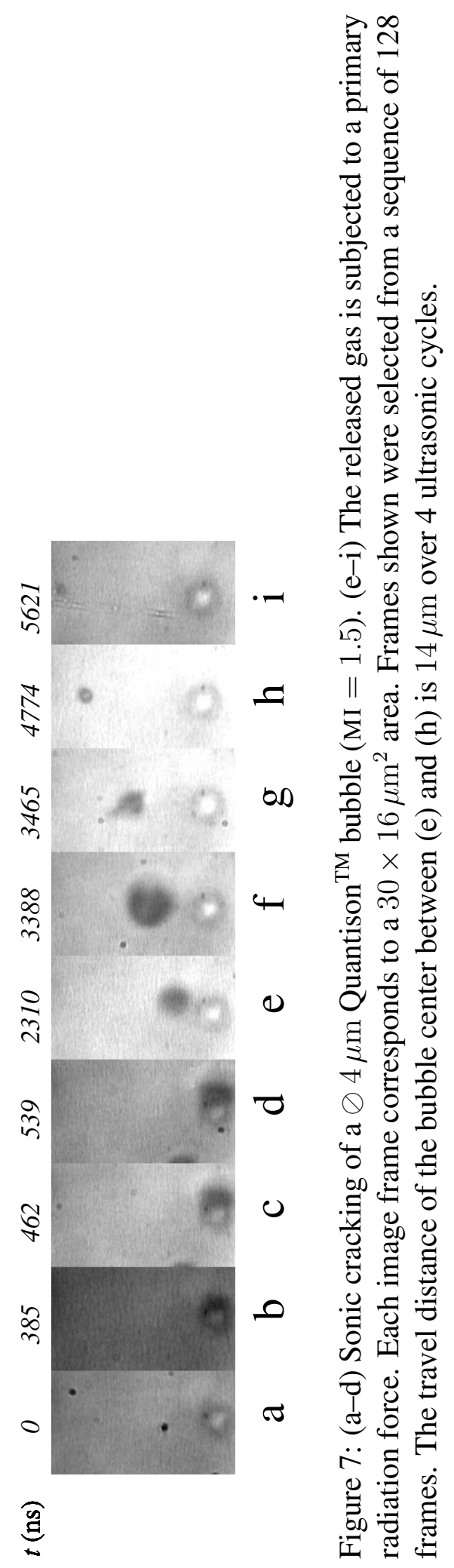



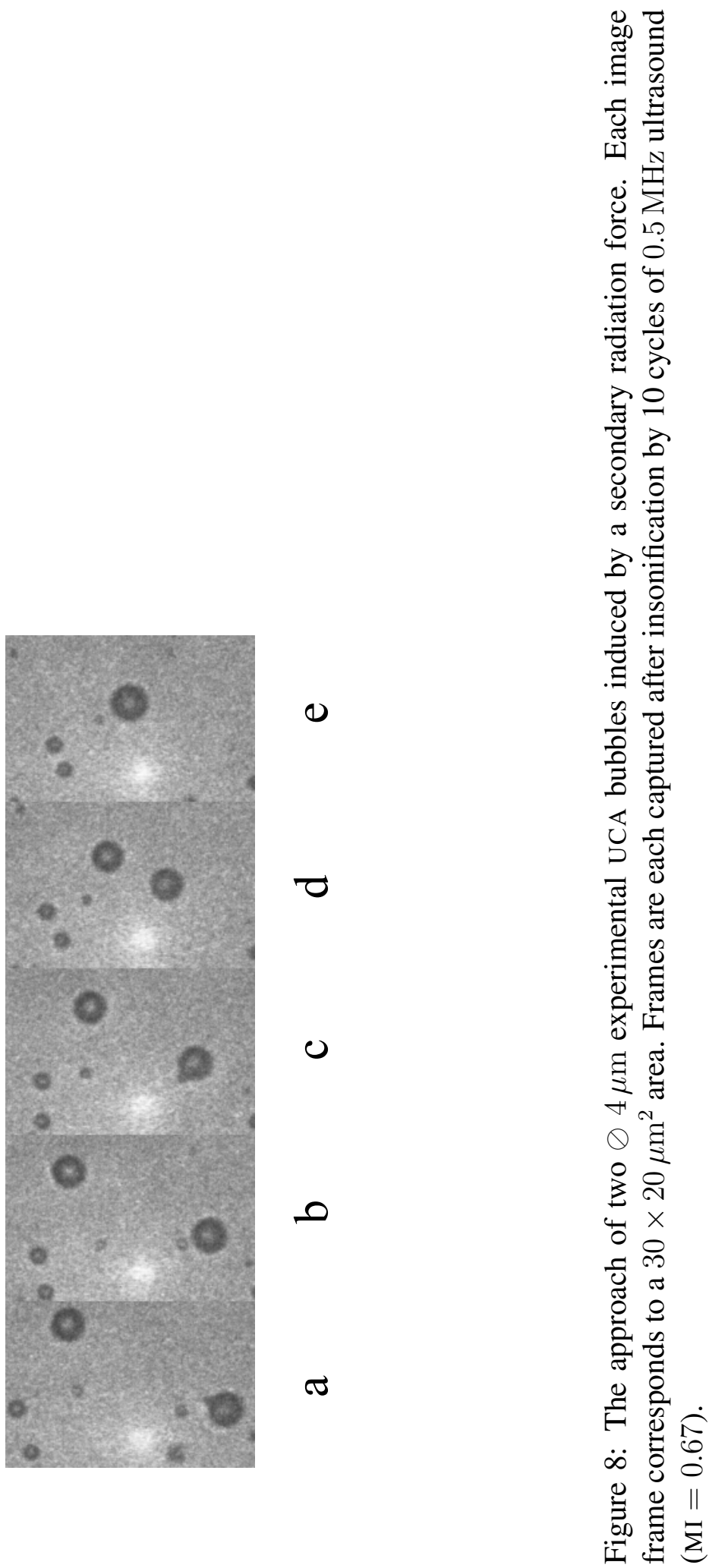


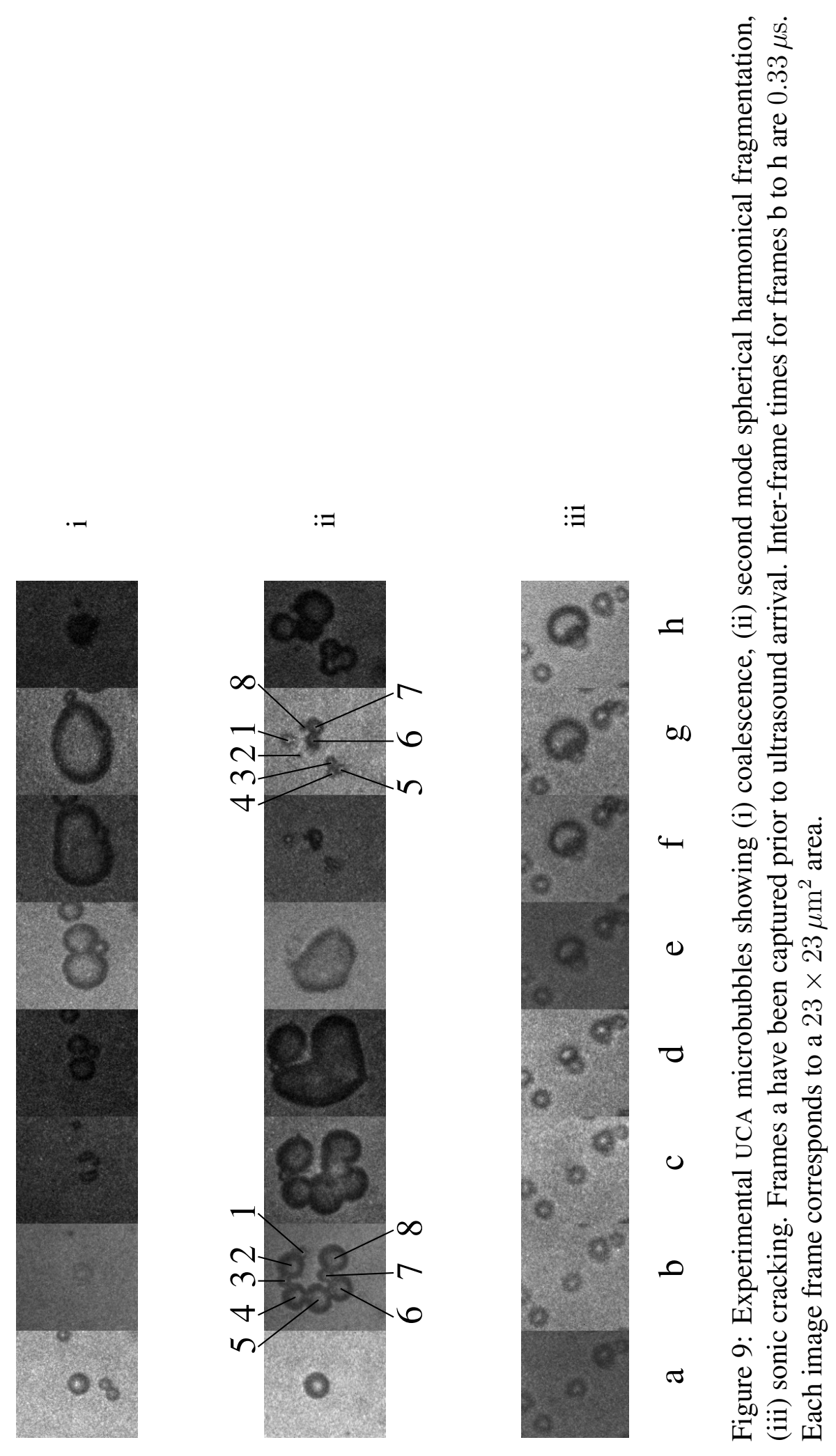




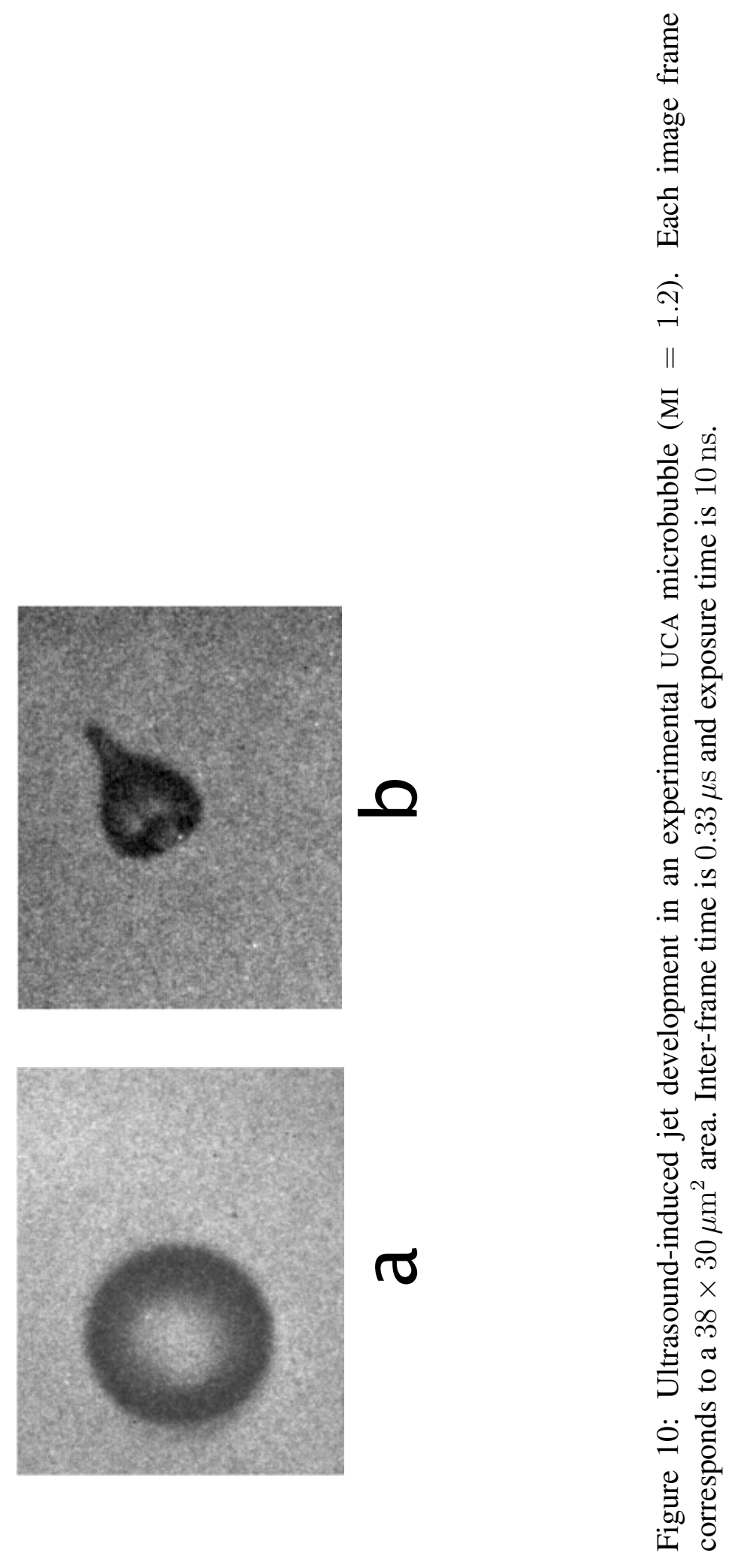

